\title{
Mallory-Weiss tear as a rare complication during esophageal endoscopic submucosal dissection
}

\author{
Jing Wen ${ }^{1}$, Jing Yang ${ }^{2}$, Zhongsheng $\mathrm{Lu}^{2}$, Xuqiang Bian³ ${ }^{3}$ Jin Huang ${ }^{3}$ \\ ${ }^{1}$ Department of Gastroenterology and Hepatology, Chinese PLA 261 Hospital, Beijing, China \\ 2Department of Gastroenterology and Hepatology, Chinese PLA General Hospital, Beijing, China \\ ${ }^{3}$ Department of Gastroenterology, Chinese PLA 153 Hospital, Zhengzhou, China
}

Videosurgery Miniinv 2019; 14 (1): 133-136

DOI: https://doi.org/10.5114/wiitm.2018.76088

\begin{abstract}
There are no previous reports of Mallory-Weiss tears occurring as complications during esophageal endoscopic submucosal dissection (ESD). We present 3 cases of Mallory-Weiss tears occurring during treatment of superficial squamous cell neoplasms through ESD. Carbon dioxide was used for air insufflation, and the patient was adequately sedated without retching or struggling during the operation. No significant bleeding from the lesion was observed during ESD; however, active bleeding caused by the Mallory-Weiss tear was identified endoscopically. Bleeding was controlled by closure of the tear with metal clips or repeated flushing with adrenaline saline. Hemorrhage did not recur until completion of the operation. Therefore, during the operation, it is necessary to maintain adequate depth of anesthesia and reduce the operation time, and also reduce air and water injections, to avoid occurrence of such complications.
\end{abstract}

Key words: Mallory-Weiss tear, complication, endoscopic submucosal dissection.

\section{Introduction}

Endoscopic submucosal dissection (ESD) is considered to be an advanced technique in therapeutic endoscopy that has replaced surgery in clinical practice. Despite the good prospects for this technology, some limitations do exist, namely the complications of hemorrhage and perforation [1-3]; other complications have been rarely reported. Hongou et al. [4] reported one case in which a Mallory-Weiss tear occurred during gastric ESD. However, there are no prior reports of Mallory-Weiss tears occurring as complications during esophageal ESD. This study analyzed 3 cases of Mallory-Weiss tears occurring during treatment of superficial squamous cell neoplasms through ESD at our hospital. In order to reduce complication risks, here we explore the causes and describe the treatment strategies.

\section{Case reports}

\section{Case 1}

A 52-year-old male patient was hospitalized because of retrosternal pain. On chromo-endoscopy iodine staining, 0-Ilb superficial esophageal cancer was detected, and the lesion was $28-30 \mathrm{~cm}$ from the incisors, accounting for about half of the lumen (Photo 1 A). Lymphatic metastasis was not detected on computed tomography. The patient consented to ESD treatment. During the operation, the patient was in a lateral position; general anesthesia with endotracheal intubation was administered after evaluation by an anesthesiologist before the operation. Routine monitoring was performed to measure blood pressure, heart rate, pulse, and oxyhemoglobin saturation. Intramuscular injection of $0.5 \mathrm{mg}$ atropine was administered before anesthesia; drugs used

\section{Address for correspondence}

Jin Huang, Department of Gastroenterology, Chinese PLA 153 Hospital, 602 Zhengshang Road, Zhongyuan District, Zhengzhou 450000, China, phone: +86 37160653761, fax: +86 37160653761, e-mail: docjinhuang@126.com 
for anesthesia induction included $0.3 \mathrm{mg}$ fentanyl, $2 \mathrm{mg}$ midazolam, target-controlled input of $2.5 \mathrm{\mu g} / \mathrm{ml}$ propofol, and $0.2 \mathrm{mg} / \mathrm{kg} 1.5$ cisatracurium besilate. Endotracheal intubation was performed after successful induction of anesthesia. Maintenance medication included $1.5 \mu \mathrm{g} / \mathrm{ml}$ propofol and $6-8 \mu \mathrm{g} /$ $\mathrm{ml}$ remifentanil. During treatment, the patient received continuous oxygen inhalation and underwent electrocardiograph monitoring, in addition to blood pressure and oxyhemoglobin saturation monitoring. During the operation, $\mathrm{CO}_{2}$ was used for air inflation.

Fifty minutes into the operation, the patient coughed repeatedly, and bright red blood was found in the lower esophagus. After flushing with normal saline, a mucosa tear, about $2 \mathrm{~cm}$ in length, was found in the greater curvature of the gastric cardia mucosa with continuous hemorrhage (Photo $1 \mathrm{~B}$ ). Metal clips were used to close the wound immediately (Photo $1 \mathrm{C}$ ). Fresh blood was not found after repeated flushing. The operation was carried out to completion. Fasting was required for the patient for 1 day, while a liquid diet was allowed 2 days later with gradual transition to solid food. Within three days after the operation, a proton pump inhibitor was intravenously administered to reduce gastric acid; it was subsequently taken orally. A week later, a residual metal clip was found on reexamination through gastroscopy, but no hemorrhage was observed.

\section{Case 2}

A 62-year-old male patient was hospitalized because of choking with sobs after deglutition. Endoscopic examination showed 0-Ilc superficial esophageal carcinoma. The lesion was $24-25 \mathrm{~cm}$ from the incisors, and accounted for about $1 / 3$ of the lumen. Endoscopic submucosal dissection was conducted for the management of early esophageal cancer under general anesthesia with endotracheal intubation. $\mathrm{CO}_{2}$ rather than room air was used for insufflation.

Fifty-five minutes into the operation, a small amount of dark red blood was found in the gastric cavity coincident with inspiration and water absorption. After flushing with normal saline, a longitudinal mucosal tear less than $2 \mathrm{~cm}$ in length was found in the antetheca of the cardia. Hemorrhage stopped after repeated flushing with $1: 10,000$ adrenaline saline solution. Hemorrhage did not occur again during the operation. An intravenous proton pump inhibitor drip was administered for 3 days; thereafter, it was taken orally. On the day of the operation, abrosia was required for the patient, while a liquid diet was allowed 2 days later, with a gradual transition to soft food. There was no alimentary tract hemorrhage at a 1-month follow-up visit after discharge.

\section{Case 3}

A 60-year-old male patient was hospitalized because of retrosternal pain. Endoscopic examination revealed a superficial squamous cell carcinoma, accounting for half of the lumen, and $34-36 \mathrm{~cm}$ from the incisors. Endoscopic submucosal dissection was performed to manage the early esophageal cancer under general anesthesia with endotracheal intubation. Sixty-five minutes into the operation, the mucosa of the lesion was completely dissected,

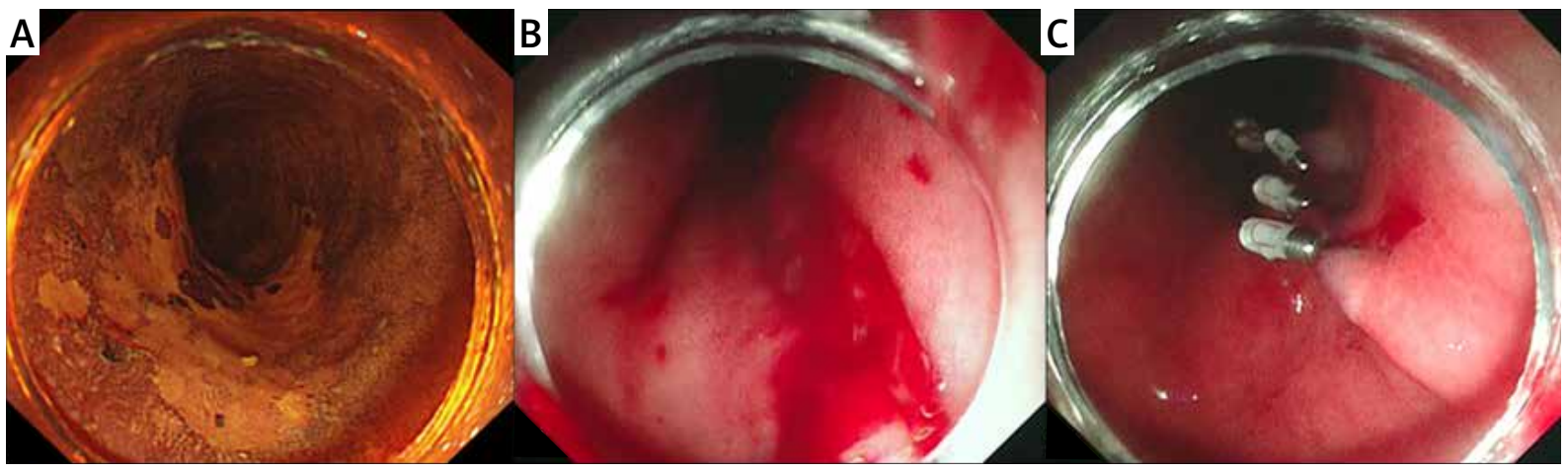

Photo 1. A - Esophageal squamous carcinoma extending up to $1 / 2$ of the circumference of the esophageal lumen, $\mathbf{B}$ - mucosa tear about $2 \mathrm{~cm}$ in length at the greater curvature of the gastric cardia with continuous disemboguing of blood, $\mathbf{C}$ - closure of Mallory-Weiss tear with metal clips 
and the patient suffered repeated hiccups. The dosages of the muscle relaxant and depressant were increased by the anesthetist to deepen the anesthesia. When the hiccups ceased, a large amount of dark red blood was found during inspiration and water absorption in the gastric cavity. The bleeding section was checked on gastroscope reversal, and showed a longitudinal mucosal tear, less than $1 \mathrm{~cm}$ in length, on the lesser curvature side of the gastric cardia with a little errhysis. Hemorrhage stopped after repeatedly flushing with 1 : 10,000 adrenaline saline solution. Hemorrhage did not occur from the mucosal tear again during the operation.

This study was conducted in accordance with the Declaration of Helsinki and with approval from the Ethics Committee of Chinese PLA 153 Hospital. Written informed consent was obtained from all participants.

\section{Discussion}

A Mallory-Weiss tear is characterized by longitudinal mucosal lacerations in the distal esophagus and proximal stomach. These tears usually occur secondary to a sudden increase in intra-abdominal pressure. Precipitating factors include vomiting, straining for stool or lifting, epileptic convulsions, coughing, hiccups under anesthesia, and closed chest massage $[5,6]$. In the endoscopy center, there have been about 500 cases of gastric ESD, and cardia mucosa tears have not occurred among these cases. There have been about 400 cases of esophageal ESD, in 3 of which $(0.75 \%)$ there occurred a cardia mucosa tear. Undoubtedly, a cardia mucosa tear is a rare complication of ESD.

There are two possible reasons for the occurrence of this rare complication. First, coughs and hiccups increase intra-abdominal pressure, resulting in a cardia mucosa tear. A cardia mucosa tear often occurs with vomiturition, emesis, and even hiccups [7, 8]. The sudden increase of intra-abdominal pressure causes pressure damage to the gastroesophageal junction. To avoid over-inhibition in the respiratory and circulatory functions caused by deep anesthesia, anesthetists always keep the patients in a superficial and moderate depth of sedation. If the depth of sedation is too superficial and there is a large amount of sputum stimulation, the patient may suffer from a frequent cough, bucking, and hiccups, which may directly lead to increased intra-abdominal pressure and a cardia mucosa tear. Second, tears may be caused by injection into the gastric cavity of an excessive amount of air and water that is not retracted in a timely manner. The esophagus has a narrow lumen and a thin wall, and is significantly affected by respiratory fluctuations and cardiac pulsation. During an operation, the surgeon may continuously inject air to expand the esophagus and expand the operation area, and repeatedly flush the operation area with water to ensure a clear operating field. Most water and air injections are deposited into the gastric cavity, which may increase the intragastric pressure and increase the risk for a cardia mucosa tear. Maeda et al. [9] reported that during gastric ESD, compared with injection of air, injection of $\mathrm{CO}_{2}$ might reduce residual gas in the alimentary canal. However, there are still reports of cardia mucosa tears occurring with injection of $\mathrm{CO}_{2}$, and the rate is reduced in comparison to that observed with injection of air [10].

To avoid these complications, the operation should cease once a patient experiences a hiccup, cough, or other symptoms, to reduce stimulation. An inhaled anesthetic, such as propofol and fentanyl, should be used to deepen anesthesia. Anesthetists should clear respiratory secretions immediately to maintain an appropriate depth of anesthesia. During ESD procedures, the maintenance of optimal levels of sedation is critical to minimize the risk of perforation and other procedure-related complications. $\mathrm{CO}_{2}$ insufflation should be used, and the operators should reduce the use of air and water injections. Timely entrance into the gastric cavity, which attracts air and liquid into the cavity and reduces intragastric pressure, also reduces the risk of cardia mucosa tear.

\section{Conclusions}

This report presents 3 cases of cardia mucosa tears occurring during ESD. There may be a certain correlation between coughs and hiccups, as well as operation techniques, in terms of the risk of cardia mucosa tears. Therefore, during the operation, it is necessary to maintain an adequate depth of anesthesia and reduce the operation time, in addition to reducing air and water injections, to avoid the occurrence of such complications. 


\section{Acknowledgments}

Jing Wen and Jing Yang contributed equally to this work.

This work was supported by Military Medical Science and Technology Youth Training Project No. 13QNP034.

\section{Conflict of interest}

The authors declare no conflict of interest.

\section{References}

1. Oyama T, Tomori A, Hotta K, et al. Endoscopic submucosal dissection of early esophageal cancer. Clin Gastroenterol Hepatol 2005; 3 (7 Suppl 1): S67-70.

2. Fujishiro M, Yahagi N, Kakushima N, et al. Endoscopic submucosal dissection of esophageal squamous cell neoplasms. Clin Gastroenterol Hepatol 2006; 4: 688-94.

3. Kim M, Jeon SW, Cho KB, et al. Predictive risk factors of perforation in gastric endoscopic submucosal dissection for early gastric cancer: a large, multicenter study. Surg Endosc 2013; 27: 1372-8.

4. Hongou H, Fu K, Ueyama H, et al. Mallory-Weiss tear during gastric endoscopic submucosal dissection. World J Gastrointest Endosc 2011; 3: 151-3.

5. Weaver DH, Maxwell JG, Castleton KB. Mallory-Weiss syn drome. Am J Surg 1969; 118: 887-92.

6. Penston JG, Boyd EJS, Wormsley KG. Mallory-Weiss tears oc curring during endoscopy: a report of seven case. Endoscopy 1992; 24: 262-5.

7. Sugawa C, Benishek D, Walt AJ. Mallory-Weiss syndrome. A study of 224 patients. Am J Surg 1983; 145: 30-3.

8. Brown JD. Hiccups: an unappreciated cause of the Mallory Weiss syndrome. Am J Med 2015; 128: e19-20.

9. Maeda Y, Hirasawa D, Fujita N, et al. A prospective, randomized, double-blind, controlled trial on the efficacy of carbon dioxide insufflation in gastric endoscopic submucosal dissection. Endoscopy 2013; 45: 335-41.

10. Takada J, Araki H, Onogi F, et al. Safety and efficacy of carbon dioxide insufflation during gastric endoscopic submucosal dissection. World J Gastroenterol 2015; 21: 8195-202.

Received: 29.03.2018, accepted: 10.05.2018. 